\title{
L’École de génie de Chicoutimi : oeuvre des Maristes (1948 - 1969)
}

Jean-Guy Genest

Volume 42, 1975

URI : https://id.erudit.org/iderudit/1007234ar

DOI : https://doi.org/10.7202/1007234ar

Aller au sommaire du numéro

Éditeur(s)

Les Éditions Historia Ecclesiæ Catholicæ Canadensis Inc.

ISSN

0318-6172 (imprimé)

1927-7067 (numérique)

Découvrir la revue

Citer cet article

Genest, J.-G. (1975). L’École de génie de Chicoutimi : oeuvre des Maristes (1948 1969). Sessions d'étude - Société canadienne d'histoire de l'Église catholique, 42 , 21-27. https://doi.org/10.7202/1007234ar

Tous droits réservés @ Les Éditions Historia Ecclesiæ Catholicæ Canadensis Inc., 1976
Ce document est protégé par la loi sur le droit d'auteur. L'utilisation des services d'Érudit (y compris la reproduction) est assujettie à sa politique d'utilisation que vous pouvez consulter en ligne.

https://apropos.erudit.org/fr/usagers/politique-dutilisation/ 


\section{L’École de génie de Chicoutimi Oeuvre des Maristes (1948 - 1969)}

En 1960, le parti de Jean Lesage prenait le pouvoir à Québec et transformait le régime scolaire de la belle province. Les mutations furent si spectaculaires qu'elles reléguèrent dans l'ombre l'œuvre accomplie par les générations précédentes. Démunies sur le plan financier, mal épaulées, parfois entravées par l'autorité gouvernementale, celles-ci n'en ont pas moins manifesté un dynamisme, un souci d'adaptation remarquables.

L'École de génie de Chicoutimi (1948-1969) est un bel exemple d'adaptation aux problèmes d'une région particulière. Après avoir retracé l'historique de cette maison d'enseignement, nous montrerons le rôle qu'y ont joué les Maristes, ces hommes d'Église qui dispensent l'enseignement au Saguenay depuis plusieurs décennies.

\section{I. - Historique de l'Ecole de génie}

L'École de génie de Chicoutimi connut des débuts modestes. À sa fondation en 1948, une douzaine d'étudiants s'y inscrivirent. Le programme était, à une virgule près, celui de la première année de l'École Polytechnique. Celle-ci assumait la direction de l'enseignement.

Les cours se donnaient dans l'immeuble de l'école secondaire ${ }^{1}$. Ils y occupaient un seul local. Celui-ci servait à la fois pour les cours, les études, les récréations et même pour le dîner des étudiants qui demeuraient à l'extérieur de Chicoutimi. Comme laboratoires, on utilisait ceux de l'école secondaire, partiellement réaménagés pour répondre aux besoins des étudiants en génie ${ }^{2}$.

Sur le plan financier, c'est la Commission scolaire de Chicoutimi qui assumait les responsabilités. Les dépenses étaient d'ailleurs

1 Depuis 1929, les écoles secondaires publiques portaient le nom d'écoles primaires supérieures. (Annuaire statistique de la province de Québec).

2 Gilles Bergeron, ing., entrevue, 1er juin 1973. Jean Paul Paquet, ing., entrevue, 7 mai 1973. 
fort modestes. Elles représentaient à peine quelques sous par propriétaire, au moment de la fondation.

L'École recrutait ses étudiants dans la section dite scientifique des écoles secondaires publiques. Très peu d'étudiants venaient du séminaire local. Car un faible pourcentage des finissants de cette institution, comme de l'ensemble des collèges classiques québécois, s'orientait vers l'étude des sciences.

Après leur première année de génie à Chicoutimi, les étudiants se dirigeaient vers Polytechnique. Un petit nombre cependant s'orientaient vers Laval ou McGill. Ces institutions reconnaissaient l'enseignement donné à Chicoutimi ${ }^{3}$.

Dès le début, les étudiants de Chicoutimi obtinrent des résultats remarquables à Polytechnique et dans les autres institutions universitaires. Quelques Chicoutimiens furent même appelés à devenir professeurs à Polytechnique. Ces résultats tiennent à plusieurs causes. Les exigences d'admission à l'École de génie étaient plutôt sévères. Une fois admis, l'étudiant était suivi de près et soumis à une discipline rigoureuse. Il devait fournir une somme de travail considérable.

À Chicoutimi, les étudiants étaient tenus au courant des succès remportés par leurs aînés à Montréal. Ils étaient conscients de la valeur de la formation dispensée par l'institution saguenéenne. Dès le début de la décennie 1950, ils demandèrent à leurs professeurs d'établir la $2^{\mathrm{e}}$ année à Chicoutimi ${ }^{4}$. Consultée à ce sujet, Polytechnique était prête à reconnaître cette deuxième année et à recevoir ensuite les étudiants en troisième 5 .

Restait à régler la question financière. La commission scolaire demanda l'aide de Québec. On lui répondit que ces cours n'étaient pas du ressort d'une commission scolaire. Même si les professeurs étaient peu rénumérés, une deuxième année de sciences appliquées représentait tout de même une dépense annuelle de quelques milliers de dollars. La commission scolaire, qui manquait déjà d'espace

3 Henri Gaudefroy, directeur de Polytechnique, à Paul Joncas, doyen de la faculté d'arpentage et de génie forestier de l'Université Laval, 9 juillet 1951. - Les archives de l'École de génie de Chicoutimi sont déposées à l'Université du Québec à Chicoutimi.

4 Joseph Bonneau, secrétaire de la Commission scolaire de Chicoutimi, à M. Gosselin, directeur à l'École de génie de Chicoutimi, 17 avril 1951 .

5 H. Gaudefroy à M. Gosselin, 26 février 1951. 
pour loger sa clientèle régulière, refusa jusqu'en 1960 d'ouvrir cette $2^{\mathrm{e}}$ année. Pendant ce temps, on devait refuser des demandes d'admission et limiter les inscriptions à 35 élèves. On occupait tout l'espace disponible dans le seul local dont on disposait ${ }^{6}$.

À la fin de la décennie 1950, cependant, un déblocage se produisit. La Commission scolaire bâtit une nouvelle école secondaire et attribua des locaux assez vastes aux étudiants en génie. Cette évolution permit d'ouvrir la deuxième année de génie en septembre 1960 ?.

L'École de génie fut alors gratifiée d'une autonomie presque complète. L'institution mère, Polytechnique, avait vu les professeurs de Chicoutimi à l'œuvre depuis douze ans. Elle leur faisait désormais entière confiance et cessa d'administrer des examens de fin d'année. Polytechnique ne conserva plus qu'un droit de regard sur la promotion des étudiants de la $2^{\mathrm{e}}$ à la $3^{\mathrm{e}}$ année.

En fait, celle-ci était établie d'après les seules notes attribuées par les professeurs de Chicoutimi. Les cas litigieux étaient examinés conjointement par le directeur de l'École de génie de Chicoutimi et le directeur de Polytechnique. En accordant cette autonomie, Polytechnique avait tenu à souligner la confiance qu'elle témoignait aux professeurs de Chicoutimi ${ }^{8}$.

Au début des années 1960, les écoles secondaires publiques de la région du Saguenay, comme de tout le Québec, regorgeaient d'étudiants. Le niveau de l'enseignement s'élevait dans ces institutoins. Les professeurs se recyclaient, les écoles elles-mêmes se dotaient de bibliothèques et de laboratoires appropriés. Les étudiants des écoles publiques étaient de mieux en mieux préparés à entreprendre des études universitaires en sciences. Aussi les effectifs de l'École de génie se gonflèrent-ils rapidement. On retrouva bientôt plus d'une centaine d'étudiants dans ces deux classes de sciences appliquées. En 1966-67, les inscriptions dépassaient le cap de $200^{9}$.

De plus, on était en pleine révolution tranquille. L'argent pour l'éducation abondait. Une loi, votée par le gouvernement Barrette puis mise au point par l'administration Lesage, permettait à

6 Paul-Émile Boulet à H. Gaudefroy, 18 septembre 1954.

7 Joseph Bonneau à M. Gosselin, 15 septembre 1951.

8 J. Laurence à M. Gosselin, 21 novembre 1959.

9 P.-E. Boulet aux membres de la Commission scolaire, 7 févrer 1966. 
l'École de génie de Chicoutimi de recevoir des octrois statutaires, au prorata en nombre d'étudiants ${ }^{10}$. La question financière était réglée.

Le directeur de l'École de génie, en accord avec ses étudiants et son personnel, se hâta d'accomplir les démarches en vue d'ouvrir la $3^{\mathrm{e}}$ année. La Commission scolaire accepta de fournir les locaux et les laboratoires. Polytechnique consentit volontiers à reconnaître l'enseignement qui serait donné ${ }^{11}$. Dans ces conditions la $3^{\text {e }}$ année s'ouvrit en 1965.

À partir de cette date, l'École de génie porta le nom d'École de génie et de sciences pures. Elle se donna en même temps une vocation plus large. Tout en conservant le cours de génie comme tronc commun de son programme, elle établit certaines options qui permettaient à ses étudiants d'entrer en troisième année à la Faculté de génie forestier de Laval ou dans certains départements de sciences pures.

L'École de génie et de sciences pures répondit aussi à la demande des instituteurs désireux d'obtenir une licence d'enseignement secondaire. À cet effet, elle conclut une entente avec la faculté des sciences de l'Université de Montréal ${ }^{12}$. À l'intention de cette clientèle particulière, l'École de génie dispensa des cours le soir et le samedi. Plusieurs dizaines d'instituteurs purent ainsi poursuivre leurs études à Chicoutimi et obtenir leur licence d'enseignement.

L'École de génie poursuivit sa marche ascendante jusqu'en 1969. Le grain de sénevé était alors devenu un grand arbre. L'École disposait d'un budget de $\$ 400,000$. Elle comptait plus de deux cents étudiants ${ }^{13}$, une vingtaine de professeurs et se disposait à ouvrir une quatrième année en sciences appliquées en septembre 1969. L'Université du Québec, fondée à cette date, prit la relève et fit de l'École de génie son département de sciences pures et appliquées. C'était le plus imposant. L’Université bénéficia ainsi d'un

10 Jean-Marie Martin, directeur général des services de l'enseignement supérieur, à J. Bonneau, 29 décembre 1961.

11 J. Laurence, directeur adjoint, École polytechnique, 13 janvier 1967.

12 Henri Favre, doyen de la faculté des sciences de l'Université de Montréal, à P.-E. Boulet, 28 mars 1967.

13 L'École de génie perdit un certain nombre de ses étudiants en 1967 : l'enseignement équivalent à celui de la première de génie fut alors confié au Cegep qui ouvrait ses portes. 
personnel d'expérience, d'un directeur averti et d'une clientèle assurée. L'existence de l'École de génie fit sans doute pencher la balance pour amener l'autorité gouvernementale à créer une université à Chicoutimi.

\section{II. - Raison de la fondation de cette école de sciences à Chicoutimi}

Puisqu'il existait une école de génie à Montréal et à Québec, il y a lieu de se demander pourquoi on a voulu dispenser cet enseignement à Chicoutimi à partir de 1948.

Le séjour dans la capitale provinciale ou dans la métropole canadienne présentait plus d'un problème. La région du Saguenay était mal reliée aux deux villes universitaires. Le boulevard Talbot n'existait pas. Le trajet Chicoutimi-Québec prenait figure de véritable expédition. L'hiver, la neige, ce "redoutable obstacle à la circulation ${ }^{14}$, fermait la région sur elle-même. Un chemin de fer poussif devenait alors le seul moyen de communication.

À ce problème du transport s'ajoutait celui du financement des études. Celui-ci était crucial. Le gouvernement du Québec n'accordait ses bourses d'études qu'avec une extrême parcimonie. La famille canadienne-française, riche en enfants mais pauvre en argent, ne pouvait assumer les dépenses d'un ou plusieurs fils poursuivant des études universitaires à l'extérieur de la région. Force était pour ces jeunes gens de renoncer aux études universitaires auxquelles leur talent et leur préparation scolaire les destinaient.

Ces abandons d'études, ces pertes de talents à la grandeur de la Province, se traduisaient, d'une certaine façon, en désastre national. À l'époque, l'Université McGill produisait à elle seule plus d'ingénieurs que Laval et Polytechnique réunies. 5\% seulement des ingénieurs canadiens étaient francophones. C'est pour cet ensemble de raisons que l'École de génie fut établie à Chicoutimi.

\section{III. - Action des Maristes}

Trois groupes de personnes ont collaboré à la fondation et au maintien de l'École de génie. Les professeurs de Polytechnique ont accepté de patroner l'École, de reconnaître son enseignement et de participer à de multiples rencontres avec les professeurs de Chicoutimi. La bienveillance de Polytechnique ne s'est jamais démentie.

14 Raoul Blanchard, Le Canada français, Montréal, Fayard (Canada), 1960 , p. 43. 
Le rôle de la Commission scolaire de Chicoutimi ne fut pas négligeable. Jusqu'en 1960, elle fut seule à supporter financièrement cet enseignement. Ce faisant, elle acceptait de se placer dans l'illégalité. Les commissions scolaires n'avaient certes pas le mandat de dispenser l'enseignement universitaire.

Malgré la collaboration de Polytechnique et l'appui de la Commission scolaire, l'École de génie de Chicoutimi n'aurait jamais vu le jour sans la volonté et la détermination des instituteurs religieux, les Maristes, qui dirigeaient l'École secondaire de Chicoutimi. L'un d'eux, Majella Gosselin, avait étudié à Polytechnique. Il correspondait avec Henri Gaudefroy, secrétaire puis directeur de cette institution pendant de nombreuses années. Celui-ci rédigeait à l'époque un manuel de mathématiques. Gosselin lisait les épreuves et suggérait des retouches ${ }^{15}$. Tout au cours de ces échanges, Gosselin entretenait Gaudefroy de son projet d'établir les premières années du cours de génie à Chicoutimi.

Gaudefroy encourageait son correspondant à poursuivre son idée et l'assurait de l'appui de Polytechnique ${ }^{16}$. Majella Gosselin fit ensuite accepter l'idée par les dirigeants de sa communauté. Ceuxci promirent de fournir les professeurs nécessaires. Majella Gosselin gagna également l'inspecteur d'écoles à son projet. Puis tous deux intervinrent auprès de la Commission scolaire.

Pour faciliter l'établissement de ces cours, Majella Gosselin et ses confrères s'appliquèrent à aplanir les difficultés. Dès 1945 , ils prirent l'initiative de renforcer l'enseignement des sciences dans les classes du cours secondaire. C'est pourquoi, en 1948, ces étudiants pouvaient entreprendre sans difficultés les études universitaires en sciences appliquées. Les Maristes s'efforcèrent également de diminuer le coût de cet enseignement. Dans ce but, ils se contentèrent du maigre salaire qu'ils recevaient alors dans les écoles secondaires - environ $\$ 900$ par an - et s'astreignirent à fournir jusqu'à 20 heures de cours par semaine. Ils aménagèrent de leurs mains les laboratoires nécessaires. Pendant une quinzaine d'années, ils accomplirent eux-mêmes le travail de secrétariat sans aucune rénumération et sans dispense d'heures d'enseignement. Jusqu'au développement vertigineux que connut l'école, pendant la décennie

15 Henri Gaudefroy à Majella Gosselin, 14 janvier 1947.

16 H. Gaudefroy à M. Gosselin, 5 mai 1948.

- Majella Gosselin, M.S., fut directeur de l'École de génie de 1948 à 1954 et de 1956 à 1965. 
1960, les Maristes assumèrent presque seuls la tâche de l'enseignement.

En diminuant ainsi le coût de cet enseignement, les Maristes permettaient à la Commission scolaire de maintenir les frais de scolarité à un niveau abordable pour les étudiants défavorisés sur le plan monétaire. Les frais de scolarité furent excessivement bas. En 1948, ils n'étaient que de $\$ 5$ par mois. Ils furent maintenus à ce niveau pendant une partie de la décennie de 1950. Ils augmentèrent quelque peu par la suite mais ils demeurèrent bien au-dessous de ceux qui étaient exigés par les universités. Les étudiants n'ayant pas à débourser pour leur pension et très peu pour leur transport, il leur était possible d'entreprendre ces études universitaires même s'ils appartenaient à des familles nombreuses, à revenus modestes et tout à fait incapables de contribuer financièrement aux études universitaires de leurs rejetons. Au total l'étudiant qui poursuivait ses études à Chicoutimi épargnait au moins $\$ 1000$ par année.

Dans l'ensemble, l'École de génie de Chicoutimi profita surtout aux fils de la classe ouvrière qui fréquentaient les écoles secondaires publiques. Au lieu de devenir techniciens dans les industries locales, ils y furent embauchés comme ingénieurs et accédèrent aux postes de direction. Aujourd'hui, au Saguenay, un grand nombre d'ingénieurs de moins de cinquante ans ont fait une partie de leur cours à l'École de génie de Chicoutimi et en sont fiers. Dans l'ensemble du Québec, plus de trois cents diplômés doivent à l'École de génie une partie de leur formation universitaire.

En créant cette œuvre et en la maintenant, les Maristes ont montré leur souci des classes populaires et leur désir de mettre l'instruction, même universitaire, à la portée de tous. Ils ont manifesté leur clairvoyance en plaçant l'accent sur la formation scientifique dans un Québec qui vouait encore un culte aux professions libérales.

Jean-Guy Genest, Professeur d'histoire Université du Québec d Chicoutimi. 